\title{
The Influence of Injected AC and DC Currents on Spontaneous Otoacoustic Emissions in the Bobtail Lizard
}

\author{
GeOfFrey A. MANLEY ${ }^{1,2}$ AND Des L. KIRK ${ }^{2}$ \\ ${ }^{1}$ Lehrstuhl für Zoologie, Technische Universität Mänchen, Garching, Germany \\ ${ }^{2}$ Department of Physiology, University of Western Australia, Nedlands, Australia
}

Received: 14 May 2001; Accepted: 20 September 2001; Online publication: 20 December 2001

\begin{abstract}
Spontaneous otoacoustic emissions (SOAEs) are accepted as being indicators of an active process in the inner ear and have been observed in all groups of land vertebrates. Previous studies have indicated that there may be more than one cellular substrate driving the active processes in different groups of vertebrates. To investigate the cellular substrate in nonmammals and to relate the findings to studies in mammalian ears, we injected AC and DC currents of amplitudes generally below $5 \mu \mathrm{A}$ into scala media of the bobtail skink Tiliqua rugosa and recorded their influences on SOAEs. Injected AC current has been shown previously to induce emissions [electrically induced otoacoustic emissions (EEOAEs)] in mammals and lizards. AC current in the appropriate frequency range entrained SOAEs but was relatively ineffective at increasing their magnitude compared with the dependence on current strength of EEOAEs generated at non-SOAE frequencies. The effects of DC current were polarity-dependent and changed over time with prolonged stimulation. Whereas positive current caused a small increase in SOAE frequency and a fall in magnitudes, negative DC current induced a decrease in SOAE frequency of more than $30 \%$, accompanied by changes in level that were strongly time-dependent. All changes were slowly reversible. The effects observed in this species of lizard strongly resemble those observed in frogs and chinchillas. The
\end{abstract}

Correspondence to: G.A. Manley, Ph.D. • Lehrstuhl für Zoologie $\bullet$ Technische Universität München • Lichtenbergstrasse $4 \bullet 85747$ Garching, Germany. Telephone: x49 892891 3660; fax: x49 892891 3674; email: geoffrey.manley@bio.tum.de similarity is interesting in view of the putatively different cellular mechanisms driving the active process in mammals and non-mammals.

Keywords: SOAE, otoacoustic emissions, lizard hearing, active process, cochlear amplifier

\section{INTRODUCTION}

In the auditory organ of vertebrates, including humans, fast mechanical amplifying systems integral to the sensory hair cells contribute to the high sensitivity and frequency selectivity of hearing at low sound levels. Evidence for such a "cochlear amplifier" has been described for all tetrapod groups (Köppl 1995; Manley and Köppl 1998; Manley 2000a). The evidence includes the presence of faint sounds (spontaneous otoacoustic emissions or SOAE) generated spontaneously by sensory cells in the inner ear and measurable in the ear canal. SOAEs are a regular feature of all lizard species so far examined (Köppl 1995; Manley 1997), and their prevalence in lizards is much higher than in standard laboratory mammals (e.g., Sun et al. 2000). It is thus not necessary to artificially induce SOAEs, e.g., through the administration of carboplatin (Hofstetter et al. 2000), and it can be assumed that SOAEs in lizards are naturally occurring phenomena. Their spectral patterns depend at least to some extent on the structure of the species' auditory papillae (Manley 1997), but, in general, their properties are remarkably similar to those of SOAE measured in mammals and in humans (Manley 2001; van Dijk et al. 1998). 
The authors (Manley et al. 2001) have also demonstrated in lizards electrically evoked otoacoustic emissions (EEOAEs) induced by AC currents applied in the scala media (Hubbard and Mountain 1983). Using this phenomenon, we presented evidence that the cochlear amplifier in lizard ears is closely linked to the hair cell bundles. This suggested that the mechanism may be homologous to that responsible for rapid movement of hair cell bundles found in vitro in hair cells in the frog sacculus and in the hearing organ of turtles (Hudspeth 1997; Ricci et al. 2000). The nature of the mechanism underlying hair cell bundle motility is, however, still unclear, and thus the mode of action of cochlear amplifier mechanisms remains of considerable interest. One mechanism suggested to contribute force to drive the stereovillar bundle involves the movement of $\mathrm{Ca}^{2+}$ ions across the transduction channels (Choe et al. 1998; Hudspeth et al. 2000).

To study further the properties of SOAEs and their relevance for elucidating the force-generating mechanisms in hair cell bundles, we examined the effects on SOAE spectra of current injection into the lizard scala media. The effects of current injection on SOAEs so far have been reported only for one frog species (Wit et al. 1989) and for the chinchilla (Sun et al. 2000).

The species we examined was the Australian bobtail skink Tiliqua rugosa (Trachydosaurus rugosus). The peripheral hearing system of this animal is perhaps the best studied of all lizard species, and SOAEs are a normal feature of this lizard's ears. The center frequencies of the SOAEs indicate that they are generated only by hair cells in the high-frequency region of the auditory papilla between about 1 and $5 \mathrm{kHz}$ (Köppl and Manley 1993).

\section{METHODS}

The experiments were carried out on five adult Australian bobtail skinks captured in the wild under license in South-Western Australia. The animals weighed between 161 and $480 \mathrm{~g}$ and were anesthetized with pentobarbital sodium and Diazepam as previously described (Manley et al. 2001). Esophageal temperature was kept at $30^{\circ} \mathrm{C}$ by a thermal blanket system and the animals were artificially ventilated at a rate and volume roughly equivalent to that of a resting animal.

To expose the ventral-lateral surface of the bony otic capsule of the inner ear, the skin and muscle layers of the lower jaw were opened lateral to the trachea. After exposure of the brain capsule, a small hole was made in the bone covering the scala vestibuli and a glass microelectrode (broken to an outer diameter (o.d.) of 3-5 $\mu \mathrm{m}$, filled with $0.2 \mathrm{M} \mathrm{KCl}$ ) was slowly advanced through the scala vestibuli and the thin vestibular membrane into the scala media. During advance, the resting DC potential, the compound action potential (CAP), and the summating potential evoked by brief tone pulses were monitored. Entry into the scala media was verified by a sudden DC shift of about $+7 \mathrm{mV}$ and an increased amplitude of soundevoked potentials. The electrode was then further advanced $0.2-0.5 \mathrm{~mm}$.

Electrical current was passed between the microelectrode and an $\mathrm{Ag} / \mathrm{AgCl}$ wire reference electrode placed under the skin of the lower jaw. The whole circuit was shielded and insulated from ground. DC electrical stimulation was achieved by passing current $(<16 \mu \mathrm{A})$ generated by a constant-current supply (AM Systems, Model 2100, Carlsborg, WA). AC currents $(<15 \mu \mathrm{A})$ were generated under software control by a high-quality PC sound card and current strength was measured from the voltage across a $100-\Omega$ resistor. AC stimuli were in the range of frequencies represented in the high-frequency area of the auditory papilla of this species, i.e., $1.0-5.0 \mathrm{kHz}$ (Köppl and Manley 1990).

A closed, calibrated sound system comprising a Bruel \& Kjær 4166 microphone coupled to a Bruel \& Kjær 2660 low-noise preamplifier was sealed onto the ear canal. The microphone output was fed either into a computer sound card for analysis and storage on disk or into a spectrum analyzer (Stanford SR 760, Sunnyvale, CA) for averaging of SOAE spectra.

Experiments were carried out under licenses from the Western Australian Department of Conservation and Land Management (SF002454) and the Animal Experimentation Ethics Committee of the University of Western Australia (98/008/C95). We also followed the guidelines of the Australian National Health and Medical Research Council.

\section{RESULTS}

Since the animals were caught in the wild, we made every effort to reduce to a minimum the number of individuals used and, where possible, took measurements from both ears. These lizards are not easy to find, and many that were seen were unsuitable since the surgical approach is generally only possible in small animals. Of the five animals used, one was used to establish the electrode approach, and, in the others, two ears failed to show SOAEs and one further ear had SOAEs that were too small. The data were thus obtained from 5 ears of 4 animals and involved 30 episodes of DC injection of different durations and amplitudes and also numerous AC injections at different frequencies and current levels. 


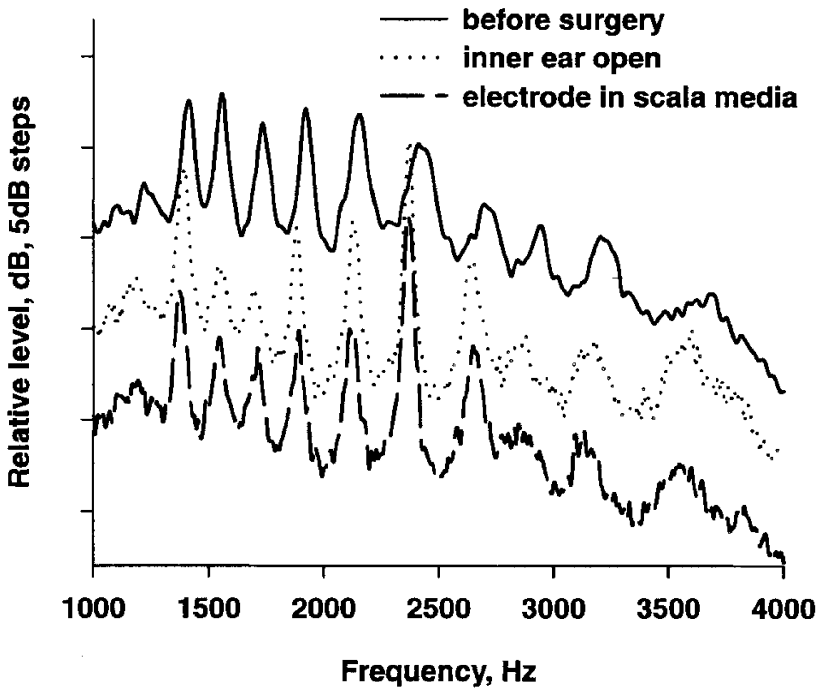

FIG. 1. Three SOAE spectra in one ear, measured using a spectrum analyzer, before the surgery (continuous line), after surgically opening the bony capsule of the inner ear (dotted line), and after placing a glass electrode in the scala media (dashed line). The three curves were shifted in the vertical axis to prevent overlap. The slight shift in frequency between the upper and lower two curves is due to a small downward adjustment in the animal's temperature.

SOAE are not damaged by the surgical procedure

Since the effects of current on SOAEs were of primary interest, it was first necessary to establish that the electrode could be placed in the scala media without irreversible damage to the SOAEs. Accordingly, SOAE spectra (200 averages) were measured following anesthesia, during and after the surgery, and during electrode penetration. While there were small changes in the SOAE spectra, often due to temperature adjustments, in no case was there a substantial loss of SOAE amplitudes (Fig. 1). We thus assume that in these ears the integrity of the cochlea was not compromised by the surgical procedures.

\section{Synchronization of SOAEs to AC current}

If we assume that SOAEs are driven by the same active process as that involved in EEOAE generation, then SOAEs should be influenced by AC current injection. This proposition was tested by averaging the ear canal sound field during AC injection, with sampling locked to the phase of the current. Using a computer sound card and custom-designed software, we collected SOAE spectra with and without injected current. Sampling/current frequency was swept from 1108 to $3919 \mathrm{~Hz}$ in steps of $39 \mathrm{~Hz}$ or over smaller frequency ranges in smaller steps. In contrast to the spectrum analyzer which detected spectral peaks regardless of

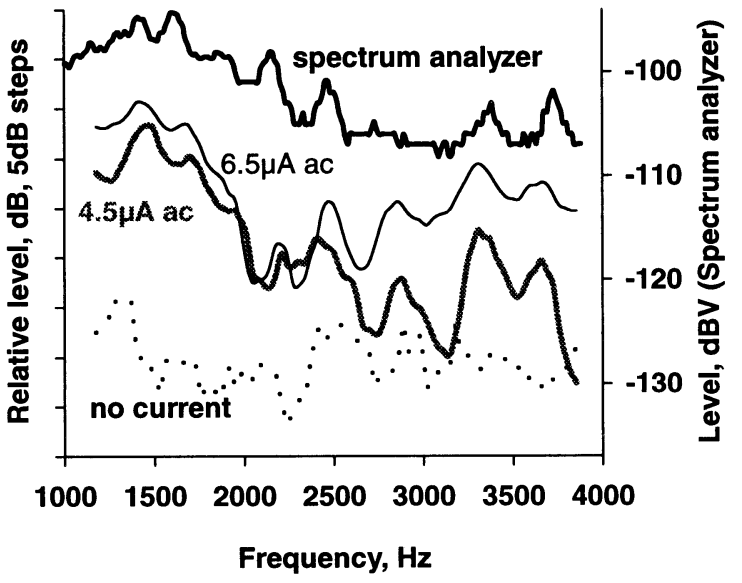

FIG. 2. A comparison of three EEOAE spectra and the SOAE spectrum in one ear. The data represented by the thick continuous black curve (right axis) was obtained using the standard spectrum analyzer with 6 small SOAE peaks. These peaks were not detected by computer spectral analysis (left axis) without current injection (dotted line), but were visible when frequency-stepped AC currents of different strengths [4.5 $\mu \mathrm{A}$ (gray line) and $6.5 \mu \mathrm{A}$ (thin continuous line)] were injected.

their phase, the computer recognized only those components that were phase-locked to the injected current. Thus, when current strength was set to zero, unsynchronized SOAEs of random phase (whose existence was established using the spectrum analyzer) were not reliably picked up by the computer. With sufficient amplitude of the swept AC current, however, phase-locked peaks at SOAE frequencies showed up in the computer spectra (Fig. 2), indicating that the AC current had entrained the SOAE-generator mechanism. The center frequency and bandwidth of these peaks did not necessarily correspond precisely to those measured by the spectrum analyzer. However, there were no clear trends in these respects. No distortionproduct otoacoustic emissions (DPOAEs) between SOAEs and EEOAEs were observed.

To verify additionally that the AC current was really entraining SOAEs, we examined SOAE and EEOAE patterns in the presence of a tone of a frequency appropriate to influence certain SOAE peaks. It is well known that tones can suppress, facilitate, and/or shift the center frequency of SOAE peaks in lizards (Manley 2000b). Would "entrained" SOAE peaks measured using the computer system show the same effects as unsynchronized SOAEs measured using the spectrum analyzer under the same conditions? A representative example is shown in Figure 3, where a SOAE spectrum with three peaks in the undisturbed condition was examined for SOAEs and EEOAEs (entrained SOAEs) in the presence of a $1.757-\mathrm{kHz}$ tone near the center peak of $1.73 \mathrm{kHz}$ and presented at different sound levels. In the SOAE spectra, the central SOAE peak was completely masked by the tone at all levels. The 


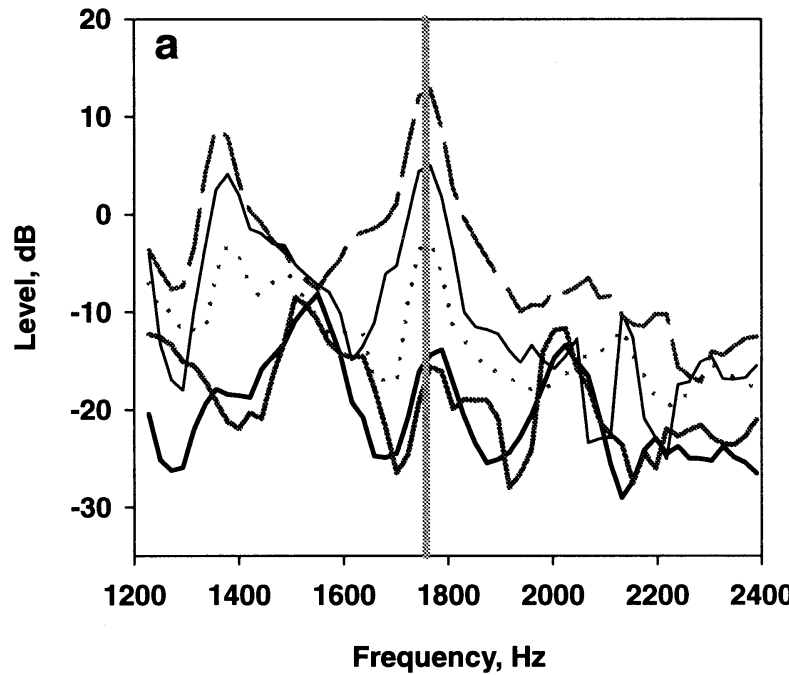

FIG. 3. An example of the effect of a tone, in this case near 1.7 $\mathrm{kHz}$, played at different attenuation levels, on EEOAEs ("entrained" SOAEs measured during swept AC injection, a) and SOAEs measured using a spectrum analyzer (b) in the same ear and over the same frequency range. The entrained SOAE peaks in the absence of the tone (a, continuous thick black line) were broader than the SOAEs measured using the spectrum analyzer (b, continuous thick black line). However, the EEOAE data show the same frequency-shifting

lowest-frequency SOAE peak was shifted to even lower frequencies as the tone level increased (Fig. 3b), a phenomenon demonstrated in earlier experiments (Köppl and Manley 1994). The entrained peak in the EEOAE data in this frequency range (center at 1.55 $\mathrm{kHz}$ ) is broader but shows the same shift to lower frequencies (Fig. 3a) as the SOAEs in Figure 3b. It even shows the small facilitation of the amplitude that occurred in the unsynchronized SOAE in the presence of the tone at $69 \mathrm{~dB}$ SPL. Both SOAE and EEOAE (entrained SOAEs) peaks were completely suppressed when the added tone reached a level of $79 \mathrm{~dB}$ SPL (not shown). The higher-frequency peak shifted up in frequency in both cases, but this was noisier in both sets of data.

Interestingly, the injection of current at frequencies at which the SOAE peaks were already present did not readily lead to an increase in the peak emission amplitude (Fig. 4a,c). At frequencies between SOAE peaks, however, there was a clear dependence of amplitude on current strength. (Fig. 4b,c). This phenomenon tended to flatten emission spectra that were measured at higher current levels.

\section{The influence of injected DC currents}

In addition to AC currents, we studied the effects of positive and negative DC currents. SOAEs were examined using the spectrum analyzer before, during, and up to one hour after DC injection (generally maximally $\pm 5 \mu \mathrm{A})$. Due to the shifting of peak frequency and

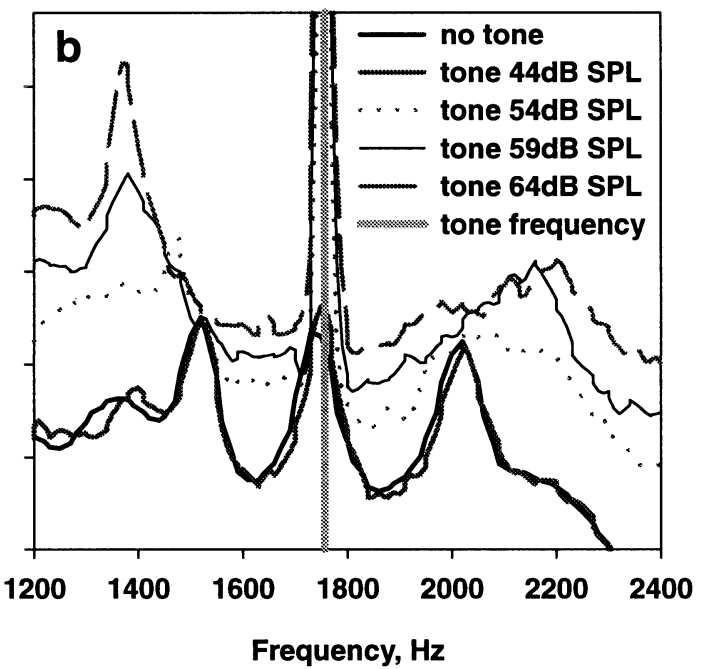

effects as do the SOAEs in the presence of the tone at different SPLs. This is clearest in the peak that initially has a peak frequency near $1500 \mathrm{~Hz}$ and shifts down in frequency as the tone level increases. This strengthens the idea that the data in a derive from SOAEs that were entrained by the current injections. The added tone was not phase-locked to the current and is thus not seen in the EEOAE traces. The bin width of the left panel data is $22 \mathrm{~Hz}$ and that of the right panel data is $15 \mathrm{~Hz}$. The legend in $\mathbf{b}$ applies to both panels.

changes in amplitude, together with peaks appearing where originally there were none, it was sometimes not easy to follow individual peaks. The time required to collect and store spectra (about $45 \mathrm{~s}$ ) compounded this problem, especially during the faster changes after the onset of the current injection. Nonetheless, consistent effects were observed, especially with regard to changes in SOAE frequency. Amplitude decreases larger than $5 \mathrm{~dB}$ of course were rarely seen, since SOAE peaks were usually smaller than $5 \mathrm{~dB}$ above the noise floor.

Negative DC induced a large downward shift (up to $31 \%$ ) in the peak frequency, often accompanied by a change in the amplitude of individual SOAE peaks (Fig. 5a). In percentage terms, the largest frequency shifts were shown by the lowest-frequency SOAE peaks (Fig. 5b), which usually rapidly became smaller and disappeared into the noise (Fig. 5c). Following current offset, these lowest-frequency peaks showed a slow recovery to near their original frequency at a rate that depended on their center frequency (Fig. 5c). During negative current injection, the amplitudes of midfrequency SOAEs increased up to $>6 \mathrm{~dB}$ initially, whereas lower- and higher-frequency SOAEs showed little increase. After about 2 minutes of current injection, the amplitudes at these midfrequencies dropped to below normal, recovering after current offset over a time period of 6-10 minutes. Recovery appeared to follow two time courses: initially rapid, then much slower.

Positive current induced a small upward shift (up 


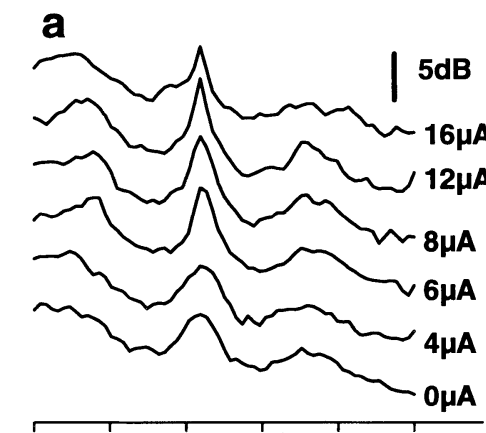

200022002400260028003000

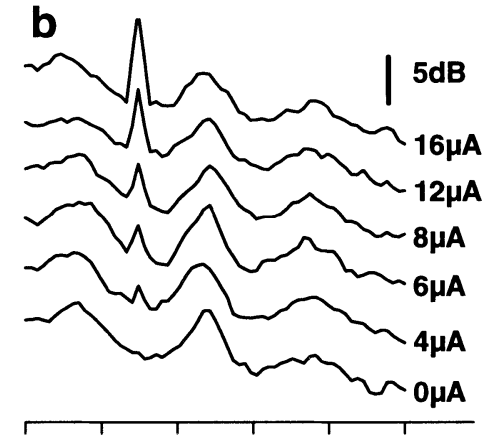

200022002400260028003000
Frequency, $\mathrm{Hz}$

FIG. 4. The ability of injected current to raise the amplitude of recorded EEOAEs depends on whether the current frequency corresponds to an SOAE peak or not. Injected current near the peak of an SOAE has little effect on the measured amplitude (a and gray line

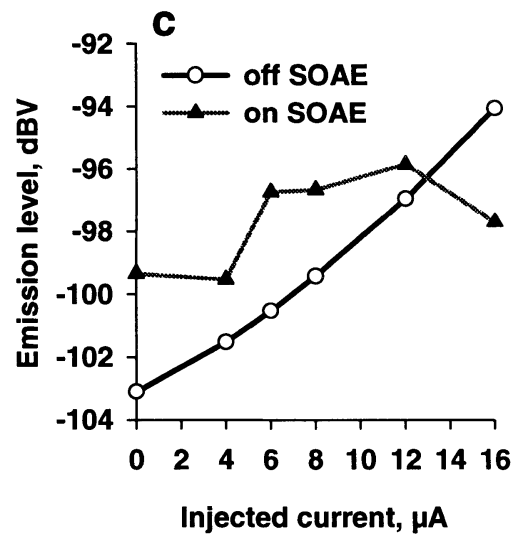

and closed triangles in $\mathbf{c}$ ), whereas current injected at a frequency remote from the SOAE peak raises amplitude almost linearly as a function of current level (b and black line and open circles in $\mathbf{c})$. The curves in $\mathbf{a}$ and $\mathbf{b}$ have been shifted in the vertical axis to avoid overlap. to $8 \%$ ) in frequencies, with most of the frequency shift occurring in the first minute (Fig. 6a,b). During positive current injection, all SOAE peaks lost amplitude; this was also especially rapid during the first minute (Fig. 6c). This was followed by no or only a small further loss until the current was turned off. Recovery following current offset was also slow.

In one case where five current densities were studied, the frequency shift tended to be larger for larger negative currents but was inconsistent for positive currents. In all cases, recovery was generally complete within 5-20 minutes after stopping the current.

In one animal, we measured the effects of DC injection on the compound action potential (CAP) in response to a $1-\mathrm{kHz}$ tone, interleaving the measurements with collection of SOAE spectral data after the current injection was over. Apart from the pretest control data, the first CAP measurement was made $15 \mathrm{~s}$ after the current was turned off. A negative current of $5 \mu \mathrm{A}$ caused a loss of at least $10 \mathrm{~dB}$ in the CAP at $1 \mathrm{kHz}$ (Fig. 7a). For a SOAE peak centered at 1.093 $\mathrm{kHz}$ (i.e., very close to the tonal stimulus frequency), a frequency shift of $27 \%$ was measured 1 minute after current was off, when this peak had reappeared in the spectrum. Positive current at the same relative time caused a frequency shift of only $3 \%$ and no measurable effect on the CAP threshold (Fig. 7b). The frequency and amplitude changes observed during negative current thus correlated with a significant effect on the responses of the hair cell-afferent fiber system.

\section{DISCUSSION}

The animal preparation we used has some advantages over the systems used to date for studying the effects of AC and DC current on SOAEs. Both Wit et al.
(1989) and Sun et al. (2000) reported results from extracochlear stimulation, which makes it difficult to estimate the true current strengths being delivered to local hair cell systems or even to know the polarity experienced by the hair cells. Since the mammalian cochlea is long and spirals, the strength and polarity of the current applied with each stimulus will be a complex function of the relative locations of the hair cells and the electrodes. Even using a glass pipette in the scala media, Sun et al. (2000) reported, not unexpectedly, opposite effects of injected DC currents on SOAEs emanating near to or remote from the electrode location.

In bobtail lizards, the papilla is only about $2 \mathrm{~mm}$ long and straight (Köppl 1988). Our electrode placement in the scala media was no more than about 1 $\mathrm{mm}$ distant from all hair cells. Therefore, we assumed that all hair cells experienced the same current polarity and that current strength did not vary greatly over the length of the papilla. In addition to this advantage, lizard ears usually show SOAE spectra with a number of peaks (unlike frogs and laboratory mammals), allowing the observation of differences between SOAEs of different center frequency.

It might be argued that the EEOAEs we observed could be the result of acoustic effects induced directly by the electrode, which might stimulate hair cells acoustically, rather than being an electrical effect on hair cells. However, the fact that EEOAEs in lizards and their acoustic modulation by low-frequency tones are susceptible to ototoxic drugs and physiological insult (Manley et al. 2001) is not consistent with this explanation. Those data indicate rather that the electrical current is directly affecting the active process in the hair cell bundles.

Although the SOAE peaks entrained by AC stimulation often differed slightly from the unsynchronized 


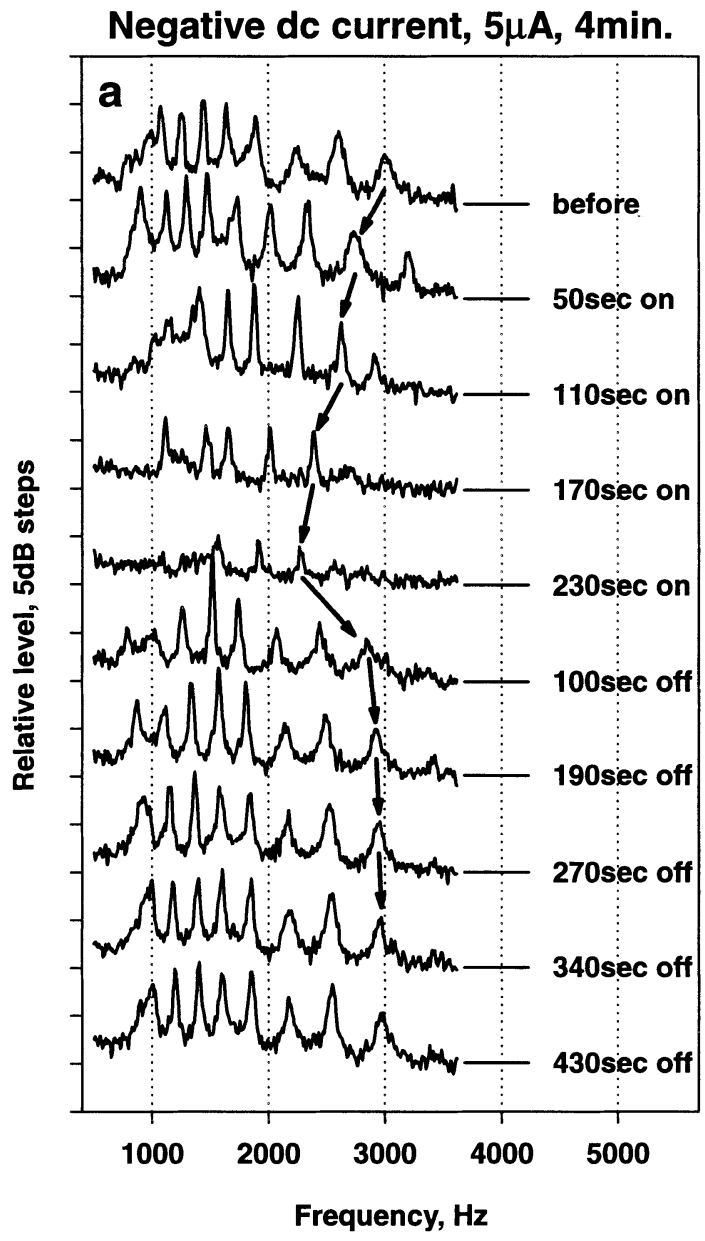

FIG. 5. An example of the effects of injecting $5 \mu \mathrm{A}$ negative direct current for 4 minutes and the start of the recovery period. a SOAE spectra measured using the spectrum analyzer before, during, and at various times after current offset (top to bottom). To avoid overlap, the successively collected spectra are shifted down in $10 \mathrm{~dB}$ steps. The arrows are a visual aid to follow the frequency shift of an individual peak. b,c An analysis of the data, quantifying the changes in

SOAEs in both center frequency and peak width (Fig. 2), the demonstration that SOAEs can be synchronized to an electrical stimulus suggests that, in lizards, the same motor system drives the SOAEs and the EEOAEs. The AC current synchronized the phase of existing hair cell oscillations to the phase of the current but did not markedly increase their magnitude. This suggests that SOAEs represent oscillations that are already near saturation for the motor system of the active hair cells. This is what would be expected from the behavior of a limit-cycle oscillator working near its peak output. At frequencies between SOAE peaks, the amplitude of the EEOAEs rose more steeply with increases in the
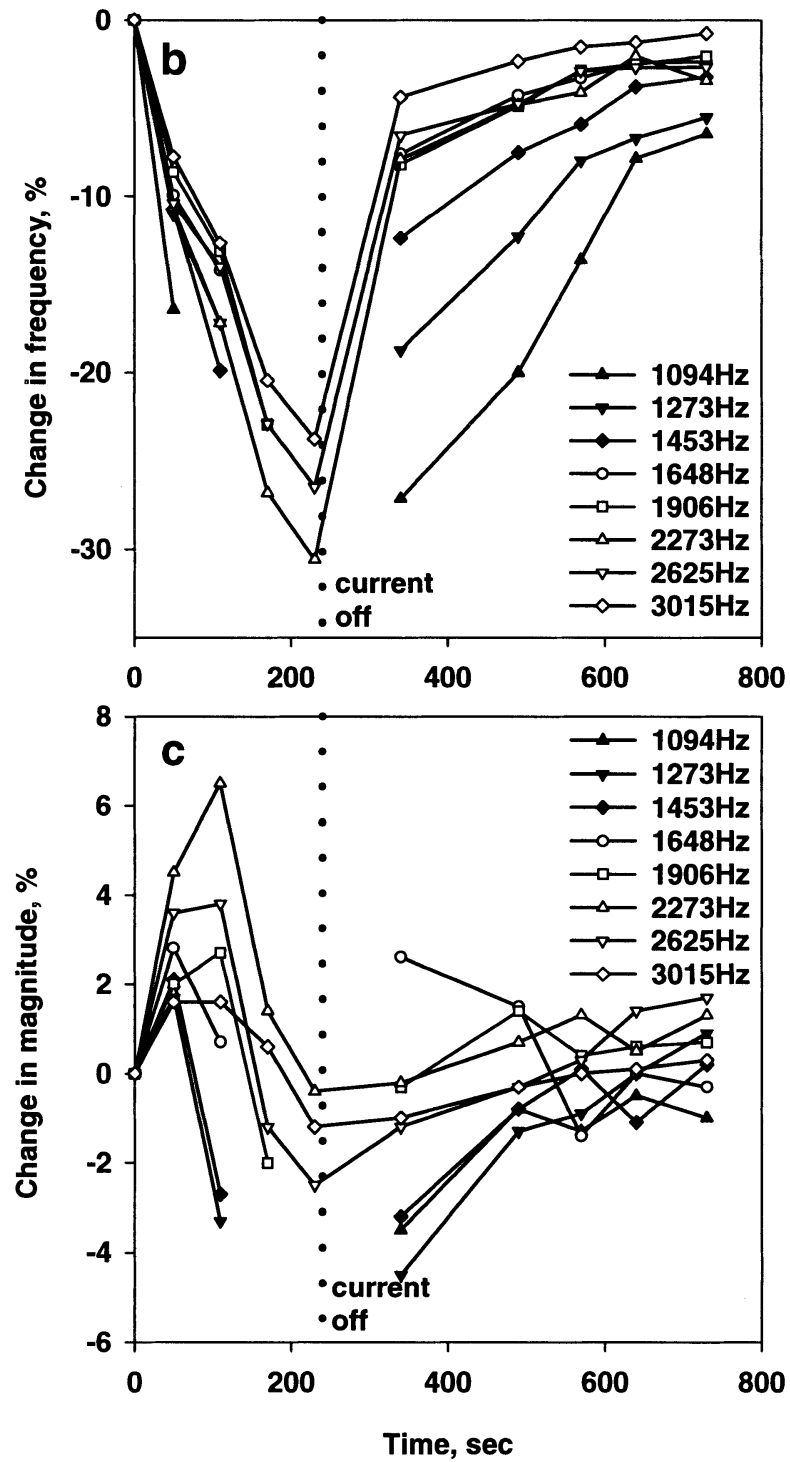

(b) frequency and (c) magnitude of SOAE peaks of different center frequency as a function of time during and after the injection. The effect of this negative current was large downward shifts in frequency (b). On a hertz scale, the frequency shifts for higher-frequency SOAE are much larger than those of lower-frequency SOAEs. Negative current initially caused an increase followed by a decline in magnitudes $(\mathbf{c})$.

applied current. This is an important point, since it suggests that, with narrowband AC stimulation, the current mainly affects the hair cells that are tuned to that particular narrow frequency band. At frequencies where hair cells are not oscillating as groups, the current possibly leads to entrainment of closely spacedperhaps previously independently vibrating-hair cells, whose coordinated oscillations then appear as an EEOAE.

In the chinchilla, distortion-product OAEs (DPOAEs), resulting from interactions between SOAEs and EEOAEs, were reported (Sun et al. 2000). We failed to observe such DPOAEs, but their absence 


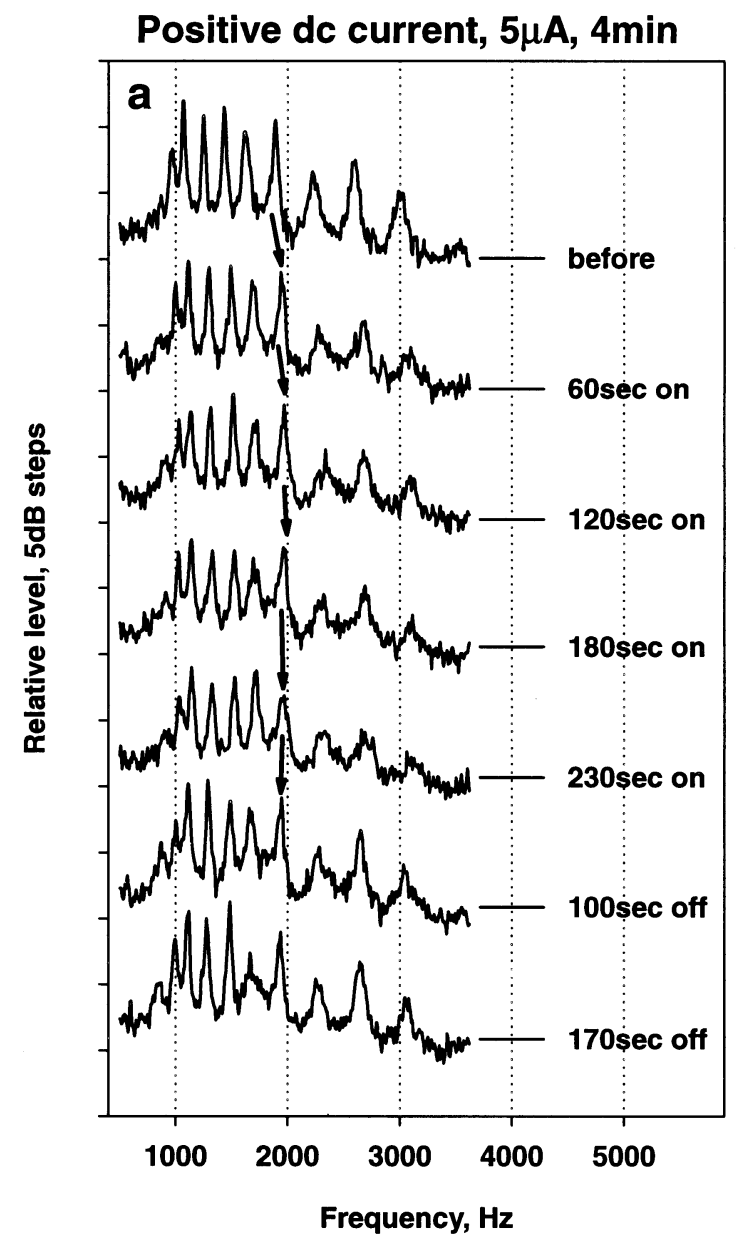

FIG. 6. An example of the effects of injecting $5 \mu \mathrm{A}$ positive direct current for 4 minutes and the start of the recovery period in the same ear as in Figure 5. a SOAE spectra measured using the spectrum analyzer before, during, and at various times after current offset (top to bottom). To avoid overlap, the successively collected spectra are shifted down in $10 \mathrm{~dB}$ steps. The arrows indicate the frequency shift of an individual peak. b,c An analysis of the data in a, quantifying

is almost certainly due to the fact that both SOAEs and DPOAEs are generally quite small in lizards, and the SOAEs we report here were certainly well below the levels often observed in mammals. The absence of DPOAEs is thus not necessarily an indication of some fundamental difference between lizard and mammal systems. DPOAEs between two introduced tones are consistently observed in lizards and have been described in some detail (Köppl et al. 1993; Manley and Köppl 1993; Taschenberger et al. 1995).

The interpretation of the effects of injected currents depends, of course, on assumptions about the basic mechanism producing SOAEs and cochlear
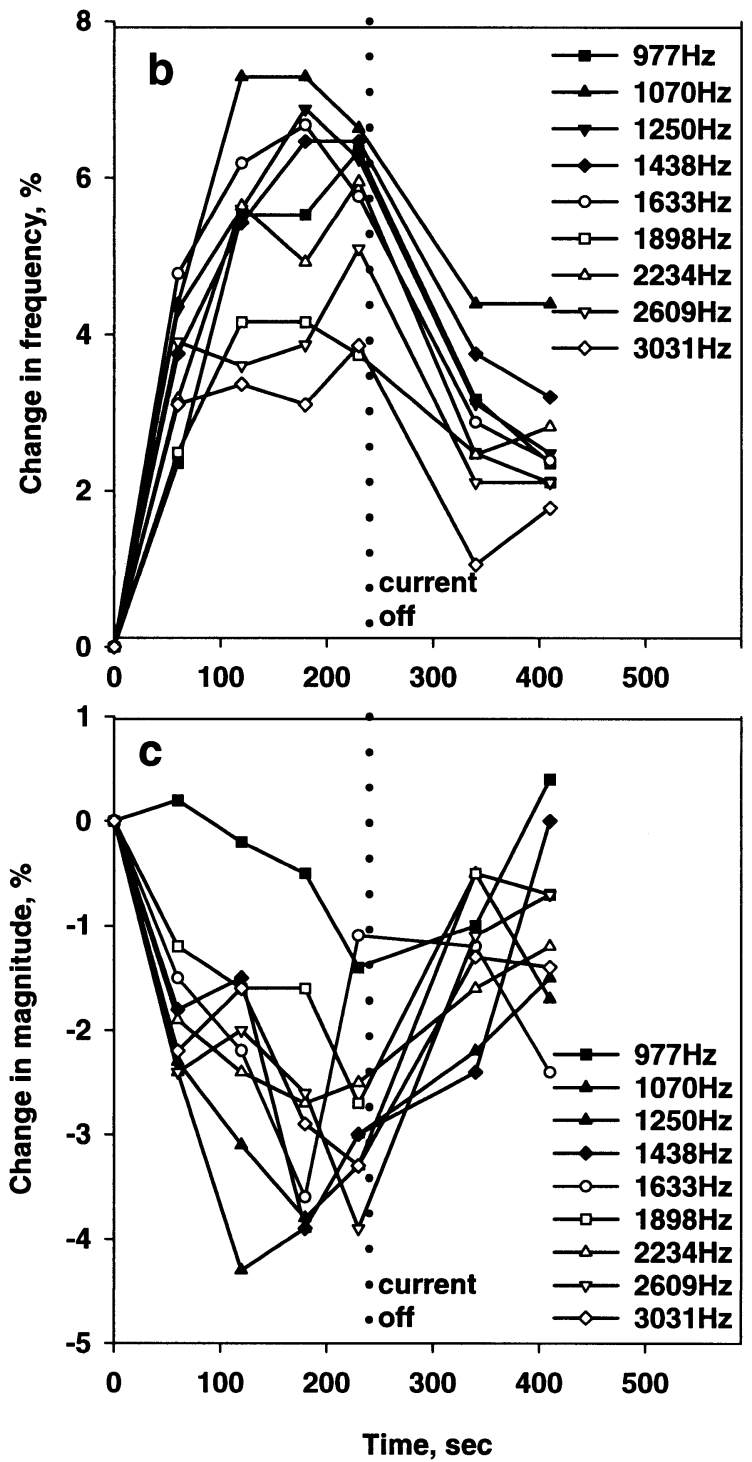

the changes in (b) frequency and (c) magnitude of the SOAE peaks as a function of time. The effect of this positive current was mainly small upward frequency shifts of SOAE (b). On a hertz scale, the frequency shifts for higher-frequency SOAEs are much larger than those of lower-frequency SOAE. In general, the positive current caused small decreases in magnitudes (c).

amplification in lizards. As indicated by our data on sound-modulated EEOAEs in the bobtail lizard (Manley et al. 2001), the active process in this species is apparently located in the hair-cell bundle. Taken together with previous data on rapid hair cell bundle motions in the frog sacculus (e.g., Benser et al. 1996) and turtle auditory papilla (Ricci et al. 2000), this suggests that the active process in all nonmammals (which lack specialized outer hair cells) is bundlebased and presumably results from a common underlying mechanism.

The only putative active-process mechanism for which there is direct evidence in nonmammals involves 

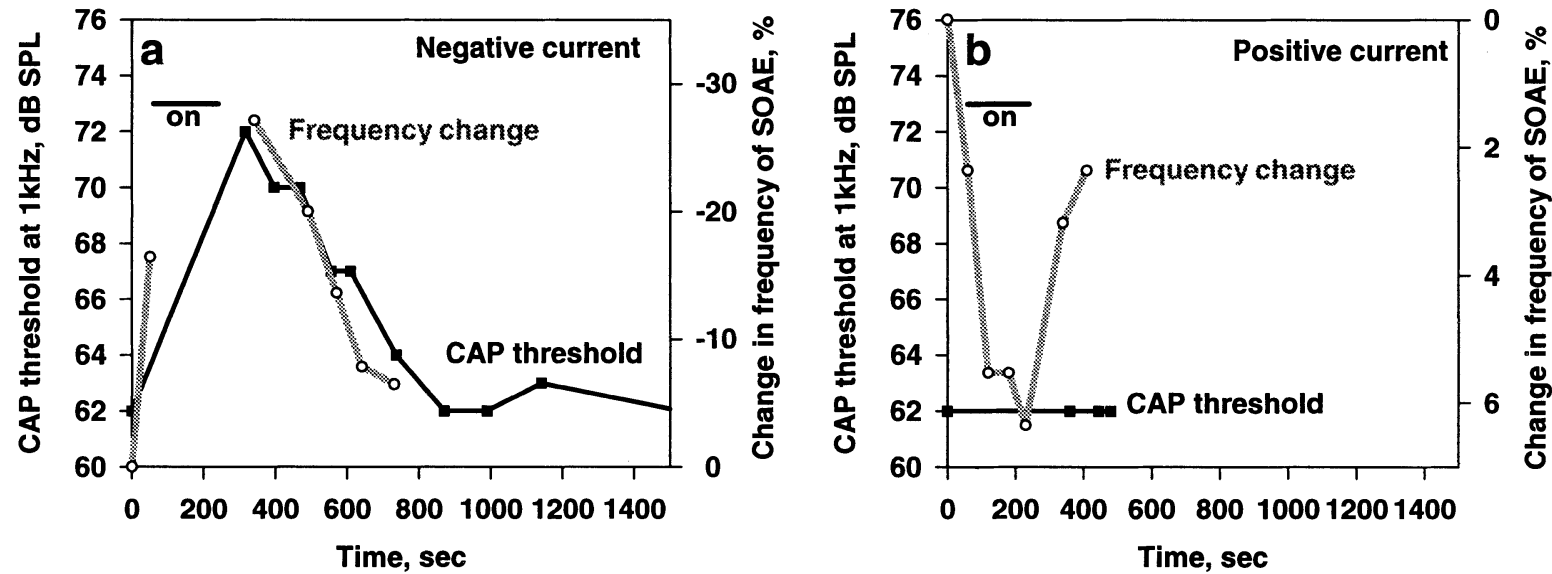

FIG. 7. The time course of the effect of negative and positive currents in the scala media on the frequency of an SOAE near $1 \mathrm{kHz}$ compared with the effect on the CAP threshold at $1 \mathrm{kHz}$. Only in the case of negative current, which has a much larger effect on the SOAE frequency (note the different frequency scales), was there a measurable change in the CAP threshold.

changes in tip-link tension and thus force exerted on the hair bundle due to the binding and unbinding of calcium ions $\left(\mathrm{Ca}^{2+}\right)$ near the transduction channels (Choe et al. 1998; Hudspeth et al. 2000). Negative current in the scala media would depolarize the hair cells' apical membranes. This would decrease the electrical drive across the transduction channels for $\mathrm{Ca}^{2+}$ ions and thus perhaps the frequency of $\mathrm{Ca}^{2+}$ bindingunbinding events. Martin and Hudspeth (1999) reported that at lower $\mathrm{Ca}^{2+}$ concentrations, which presumably also slow down the kinetics, the oscillation frequency of saccular hair cell bundles falls. In our experiments, negative current induced a large downward shift in the peak frequency of individual SOAE peaks. The two-stage recovery of the SOAE frequency may indicate that different $\mathrm{Ca}^{2+}$ stores of the cell have been depleted and are replenished at different rates. The loss of CAP threshold observed during negative current injection is difficult to interpret, but it could also be a consequence of changes in $\mathrm{Ca}^{2+}$ levels and $\mathrm{Ca}^{2+}$ stores in the hair cells.

It is interesting that the lowest-frequency SOAE peaks showed the largest percentage shifts in frequency. Their generators appear unable to sustain oscillation under current conditions, causing a substantial fall in frequency, and when their resonance frequency falls more than $30 \%$ below normal, their vibration amplitudes fall so far that the associated SOAEs disappear into the noise. Thus, we did not observe "manipulated" SOAE peaks at frequencies below those normally observed in "unmanipulated" ears (Köppl and Manley 1993). In addition, the recovery rates of low-frequency peaks were substantially slower than those of the high-frequency peaks, and in some cases more than 20 minutes were needed for full recovery.

Direct current injection and its effects on SOAEs have been described from experiments with frogs (Wit et al. 1989) and chinchillas (Sun et al. 2000). However, there were differences in current magnitudes and electrode placement between these experiments themselves, as well as with our own that make the interpretation and the comparison of the data difficult. In each case, however, the data can be interpreted such that negative current near the hair cells causes the frequency of SOAEs to fall. The fact that the frequency changes we observed are often relatively large should come as no surprise. In other circumstances, SOAEs of lizards are under poor frequency control. Under the influence of added tones, for example (frequency "pushing" and frequency "pulling"), we reported shifts in frequency of SOAE peaks of more than $300 \mathrm{~Hz}$ compared with very much smaller shifts in humans (Köppl and Manley 1994). Also, the frequency bandwidth of lizard emissions is large compared with those of mammals (Köppl and Manley 1994).

These large frequency shifts during negative current were accompanied by a drop in the sensitivity of the hearing organ as estimated from the CAP response. In mammals also, negative current at the hair cell pushed the SOAE frequency down (Sun et al. 2000) and significantly reduced the CAP sensitivity for frequencies located near the electrode (Nuttall 1985).

One fact that has become clear through our data is that shifts in the frequency and amplitude of SOAEs due to current injection do not depend on only the current strength. The effects in lizards also depended strongly on the duration of the injection, and the effect on amplitudes could even reverse polarity during longer injections (Fig. 5). Future studies of current injection thus need to take into account not only the electrode placement with respect to the target hair cells and the current strength and polarity, but also the duration of the current. Without strict control 
of these parameters, the data interpretation becomes difficult, especially in view of possible differences between putative active processes in the hair cells of mammals and nonmammals (Hudspeth 1997; Manley 2001; Sun et al. 2000).

The fact that very similar effects of DC injection on SOAEs have now been reported in frogs (Wit et al. 1989), lizards, and the chinchilla (Sun et al. 2000) is interesting in regard to consideration of the mechanisms underlying active processes in these three groups. While it is not surprising that the frog data, coming from a nonmammal, are similar to our lizard data, it would not necessarily be expected that the active process in mammals, as it is commonly understood, would show the observed similarity in the responses of SOAEs to current. It has previously been demonstrated that there are other remarkable and unexpectedly detailed similarities in the properties of nonmammalian and mammalian SOAE (Manley 2001). This generalization can now be extended to the effects of current on the hair cells, but clearly further work is necessary to clarify the uncertainties regarding the underlying mechanisms.

\section{ACKNOWLEDGMENTS}

This work was supported by grants from the Australian National Health and Medical Research Council (Project Grant No. 960566) to our late colleague Dr. Graeme Yates, who also wrote the software, and to D.L. Kirk (Project Grant No. 139003). Support also came from a grant to GAM from the Deutsche Forschungsgemeinschaft (MA 871/11-1) and a supplement to travel expenses to GAM from the Adam Haker Fonds (Stifterverband der Deutschen Wissenschaft).

\section{REFERENCES}

Benser Me, Marquis RE, Hudspeth AJ. Rapid, active hair-bundle movements in hair cells from the bullfrog's sacculus. J. Neurosci. 16:5629-5643, 1996.

Choe Y, Magnasco MO, Hudspeth AJ. A model for amplification of hair-bundle motion by cyclical binding of $\mathrm{Ca}^{2+}$ to mechanoelectrical-transduction channels. Proc. Natl. Acad. Sci. USA 95:1532115326, 1998

Hofstetter P, Ding D, SAlvi R. Induction of spontaneous otoacoustic emissions in chinchillas from carboplatin-induced inner hair cell loss. Hear. Res. 150:132-136, 2000.

Hubbard AE, Mountain DC. Alternating current delivered into the scala media alters sound pressure at the eardrum. Science 222:510-512, 1983.

HUdSPETH AJ. Mechanical amplification of stimuli by hair cells. Curr. Opin. Neurobiol. 7:480-486, 1997.

Hudspeth AJ, Choe Y, Mehta AD, Martin P. Putting ion channels to work: Mechanoelectrical transduction, adaptation, and amplification by hair cells. Proc. Natl. Acad. Sci. USA 97:11765-11772, 2000 .
KöPPL C. Morphology of the basilar papilla of the bobtail lizard Tiliqua rugosa. Hear. Res. 35:209-228, 1988.

KöPPL C. Otoacoustic emissions as an indicator for active cochlear mechanics: A primitive property of vertebrate auditory organs. In: Manley GA, Klump GM, Köppl C, (eds) Advances in Hearing Research. World Scientific Publishing Singapore, 1995, p. 207-218.

KÖPPL C, MANLEY GA. Peripheral auditory processing in the bobtail lizard Tiliqua rugosa: II. Tonotopic organization and innervation pattern of the basilar papilla. J. Comp. Physiol. A 167:101-112, 1990.

KÖPPl C, Manley GA. Spontaneous otoacoustic emissions in the bobtail lizard. I: General characteristics. Hear. Res. 71:157-169, 1993.

Köppl C, Manley GA. Spontaneous otoacoustic emissions in the bobtail lizard. II: Interactions with external tones. Hear. Res. 72:159-170, 1994

KöPpl C, MANLEy GA, Johnstone BM. Acoustic distortion products in the ear canal of the bobtail lizard. I: General characteristics. J. Acoust. Soc. Am. 93:2820-2933, 1993.

MANLEYGA. Diversity in hearing-organ structure and the characteristics of spontaneous otoacoustic emissions in lizards. In: Lewis ER, Long GR, Lyon RF, Narins PM, Steele CR, (eds) Diversity in Auditory Mechanics. World Scientific Publishing Singapore, 1997, p. 32-38.

MANLEY GA. Do non-mammals have a cochlear amplifier? In: Wada H, Takasaka T, Ohyama K, Ikeda K, Koike T, (eds) Recent Developments in Auditory Mechanics. World Scientific Publishing Singapore, 2000a, p. 499-505.

Manley GA. Otoacoustic emissions in lizards. In: Manley GA, Fastl H, Kössl M, Oeckinghaus H, Klump GM, (eds) Auditory Worlds: Sensory Analysis and Perception in Animals and Man. Wiley-VCH Weinheim, 2000b, p. 93-102.

MANLEY GA. Evidence for an active process and a cochlear amplifier in non-mammals. J. Neurophysiol. 86:541-549 2001.

MANLEY GA, KÖPPL C. Acoustic distortion products in the ear canal of the bobtail lizard. II: Suppression tuning characteristics. J. Acoust. Soc. Am. 93:2834-2844, 1993.

Manley GA, KÖPPL C. Phylogenetic development of the cochlea and its innervation. Curr. Opin. Neurobiol. 8:468-474, 1998.

Manley GA, Kirk D, Köppl C, YATES GK. In-vivo evidence for a cochlear amplifier in the hair-cell bundle of lizards. Proc. Natl. Acad. Sci. USA 98:2826-2831, 2001.

MARTIN P, HUdSPETH AJ. Active hair-bundle movements can amplify a hair cell's response to oscillatory mechanical stimuli. Proc. Natl. Acad. Sci. USA 96:14306-14311, 1999.

NUTTALL AL. Influence of direct current on DC receptor potentials from cochlear inner hair cells in the guinea pig. J. Acoust. Soc. Am. 77:165-175, 1985.

RicCi AJ, CRAWFORD AC, FETTIPLACE R. Active hair bundle motion linked to fast transducer adaptation in auditory hair cells. J. Neurosci. 20:7131-7142, 2000.

Sun W, Ding D, Reyes S, SAlvi RJ. Effects of AC and DC stimulation on chinchilla SOAE amplitude and frequency. Hear. Res. 150:137148, 2000.

Taschenberger G, Gallo L, Manley GA. Filtering of distortionproduct otoacoustic emissions in the inner ear of birds and lizards. Hear. Res. 91:87-92, 1995.

VAN DijK P, MANLEY GA, GALlo L. Correlated amplitude fluctuations of spontaneous otoacoustic emissions in five lizard species. J. Acoust. Soc. Am. 104:1559-1564, 1998.

Wit HP, van DiJK P, SEgENHOUT JM. DC injection and spontaneous otoacoustic emission frequency in the frog. Hear. Res. 41:199204, 1989 